\title{
Gemini observations of Wolf-Rayet stars in the Local Group starburst galaxy IC 10*
}

\author{
P. A. Crowther ${ }^{1}$, L. Drissen ${ }^{2}$, J. B. Abbott ${ }^{1}$, P. Royer ${ }^{3,4}$, and S. J. Smartt ${ }^{5}$ \\ ${ }^{1}$ Dept of Physics and Astronomy, University College London, Gower St, London WC1E 6BT, UK \\ 2 Département de Physique, Université Laval, and Observatoire du Mont Mégantic, Québec QC G1K 7P4, Canada \\ 3 Institut d'Astrophysique, Université de Liège, 5 av. de Cointe, 4000 Liège, Belgium \\ ${ }^{4}$ K.U. Leuven, Instituut voor Sterrenkunde, Celestijnenlaan 200B, 3001 Leuven, Belgium \\ 5 Institute of Astronomy, University of Cambridge, Madingley Road, CB3 OHA, UK
}

Received 2 January 2003 / Accepted 31 March 2003

\begin{abstract}
We present Gemini-N GMOS and CFHT MOS spectroscopy of Wolf-Rayet candidates in the Local Group dwarf galaxy IC 10 that were previously identified by Massey et al. and Royer et al. From the present spectroscopic survey, the WC/WN ratio for IC 10 remains unusually high, given its low metallicity, although none of the WC9 stars suspected from narrow-band imaging are confirmed. Our spectroscopy confirms 9 newly discovered Wolf-Rayet candidates from Royer et al., whilst spectral types of 14 Wolf-Rayet stars previously observed by Massey \& Armandroff are refined here. In total, there are 26 spectroscopically confirmed Wolf-Rayet stars in IC 10. All but one of the fourteen WC stars are WC4-6 stars, the exception being \# 10 from Massey et al., a broad-lined, apparently single WC7 star. There are a total of eleven WN stars, which are predominantly early WN3-4 stars, but include a rare WN10 star, \#8 from Royer et al. \#5 from Massey et al. is newly identified as a transition WN/C star. Consequently, the WC/WN ratio for IC10 is $14 / 11 \sim 1.3$, unusually high for a metal-poor galaxy. Re-evaluating recent photometric data of Massey \& Holmes, we suggest that the true WC/WN ratio may not be as low as $\sim 0.3$. Finally, we present ground-based finding charts for all confirmed WR stars, plus HST/WFPC2 charts for twelve cases.
\end{abstract}

Key words. galaxies: individual: IC 10 - galaxies: starburst - stars: evolution - stars: Wolf-Rayet

\section{Introduction}

Amongst Local Group galaxies, IC 10 is remarkable for its very high star formation rate, as inferred from an exceptional H II population (Hodge \& Lee 1990), large far-IR luminosity (Melisse \& Israel 1994), a non-thermal radio continuum (Yang \& Skillman 1993) and a high surface density of massive stars (Massey \& Armandroff 1995). These are direct evidence for a young, widespread burst of star formation. Indeed, IC 10 may be considered as a blue compact dwarf (Richer et al. 2001). This starburst phenomenon is also reflected in its large population of Wolf-Rayet stars, which is discussed here. IC 10 is about a factor of two smaller than the SMC, with a comparable metallicity, $\log (\mathrm{O} / \mathrm{H})=8.26$ (Garnett 1990), and so represents another rare opportunity to study individual massive stars at low metallicity.

Despite its nearby distance of only $0.6-0.8 \mathrm{Mpc}$ (Kennicutt et al. 1998; Borissova et al. 2000), the study of stellar populations of IC 10 is made difficult by its location towards the

Send offprint requests to: $\mathrm{P}$. A. Crowther,

e-mail: pac@star.ucl.ac.uk

* Figure 2 is only available in electronic form at http://www. edpsciences.org
Galactic plane, with a foreground reddening of $E(B-V)=$ 0.77-0.85 (Sakai et al. 1999; Richer et al. 2001). Massey et al. (1992) first highlighted the apparent massive star peculiarity of IC 10, suggesting the presence of 22 Wolf-Rayet stars from narrow-band photometry. Massey \& Armandroff (1995) subsequently spectroscopically confirmed 15 stars, with a highly unusual WC/WN ratio of 2, twenty times higher than the SMC (Massey \& Duffy 2001). This is puzzling, given that all other Local Group galaxies show a reasonably tight correlation of decreasing WC/WN ratio with lower metallicity. Very massive stars will evolve to the $\mathrm{WC}$ phase via the $\mathrm{WN}$ stage, whilst less massive stars might not advance to the WC stage at reduced metallicity, due to weaker winds throughout its evolution (Massey 2003).

As Massey \& Johnson (1998) have argued, an exceptionally high WC/WN ratio for IC 10 might only be explained by (i) an Initial Mass Function (IMF) skewed towards very massive stars, yet Hunter (2001) finds a normal IMF for intermediate mass stars; (ii) an exceptional $\sim 5 \mathrm{Myr}$ old galaxy wide starburst which would produce the unusual WC/WN ratio (Schaerer \& Vacca 1998); (iii) incompleteness amongst the WR population, especially amongst WN stars. It is well known that WN stars are more difficult to detect from imaging 
surveys since their emission lines are generally much weaker (e.g. Armandroff \& Massey 1985).

Subsequent to the original Massey et al. (1992) survey, Royer et al. (2001) used deep narrow-band imaging to suggest the presence of another $13 \mathrm{WR}$ stars in IC 10. Amongst these candidates, Royer et al. announced the detection of three WC9 stars. This was totally unexpected, given that such late WC subtypes stars have only previously been observed within the metal-rich regions of the Milky Way. All WC stars in the lowmetallicity Magellanic Clouds are of subtype WC4-5 or WO (Breysacher et al. 1999), a sequence that IC 10 was expected to follow. Consequently, we have obtained follow-up spectroscopy of most WR candidates in IC 10 using the Gemini-N facility multi-object spectrograph GMOS, with the remainder of other candidates observed with the Canada France Hawaii Telescope (CFHT). The present paper discusses our new observations in Sect. 2, presents a revised catalogue of Wolf-Rayet stars in IC 10 in Sect. 3. Following the completion of our study, Massey \& Holmes (2002) have recently presented a new imaging survey of IC 10, which claimed to have resolved the hitherto unusual WC/WN ratio via the discovery of many new (principally) WN stars. We discuss the present results in the light of this recent work in Sect 4.

\section{Observations}

\subsection{Spectroscopy}

Spectroscopy of WR candidates in IC 10 were obtained with GMOS (Hook et al. 2002) at the 8m Gemini-N on Mauna Kea during 21 December 2001-16 January 2002, via a joint Canadian-UK program, with principal investigators LD and PAC. In order for the necessary slit masks to be prepared, a series of images with GMOS was necessary. A series of four images, each with 100 exposures, were taken of two fields during poor seeing $\left(1-1.5^{\prime \prime}\right)$ on 20 November 2001 with the $g^{\prime}$ Gunn filter. Nevertheless, these were of sufficient quality for individual sources to be identified via comparison with William Herschel Telescope narrow-band images obtained by Royer et al. (2001).

Approximately 15 WR candidates were simultaneously observed with GMOS in each of the two overlapping fields - centred at RA = 002028 and Dec $=+591740(\mathrm{~J} 2000$, hereafter GMOS-UK) and RA = 002014 Dec $=+591825$ (GMOSCan). In all cases, a 1.0 arcsec slit and B600 grating (centred at $560 \mathrm{~nm}$ ) were used, permitting a 2 pixel spectral resolution of $5 \AA$. Total integration times of $5 \times 3600 \mathrm{~s}$ and $4 \times 3600 \mathrm{~s}$ were obtained for targets in the GMOS-UK and GMOS-Can fields, respectively. Two Wolf-Rayet stars (Massey \#5 and \#19) were common to both fields. Spare slits were used to observe sky regions, or H II regions from Hodge \& Lee (1990). GMOS consists of three $2048 \times 4608 \mathrm{EEV}$ CCDs $(13.5 \mu \mathrm{m}$ pixels), such that the longer axis of the $6144 \times 4608$ pixel focal plane is placed in the dispersion direction. (The detector array is close packed with $0.5 \mathrm{~mm}$ gap between detector imaging areas.) Pipeline Gemini specific IRAF procedures were used to reduce the datasets. An internal $\mathrm{CuAr}$ arc lamp was used for wavelength calibration, whilst a 60 s exposure of G191-B2B provided a flux calibration.

Since many of the WR candidates in IC 10 are closely spaced spatially, we were unable to observe all 28 candidates in our two overlapping GMOS fields. Consequently, we observed a small subset of candidates with MOS at the $3.6 \mathrm{~m}$ CFHT on 18 October 2001, using the $2048 \times 4500$ EEV1 CCD. Three $1200 \mathrm{~s}$ exposures were obtained with the B600 grating at moderate airmass 1.5-1.7. Slits were cut, in real time, to a slit width of $1.5^{\prime \prime}$, providing a spectral resolution of $7 \AA$. Data were reduced and extracted using standard IRAF procedures, except that wavelength calibration was achieved via FIGARO. No flux calibration was attempted for the CFHT datasets.

\subsection{Photometry}

We have adopted continuum magnitudes for IC $10 \mathrm{WR}$ candidates from Royer et al. (2001), using their dedicated filter set (Royer et al. 1998). Essentially, their c1 filter $\left(\lambda_{\mathrm{c}}=5055 \AA\right.$, $F W H M=51 \AA$ ) is close to the usual WR narrow-band $v$ filter $\left(\lambda_{\mathrm{c}}=5160 \AA, F W H M=130 \AA\right)$ defined by Smith (1968), whilst their $\mathrm{c} 2$ filter $\left(\lambda_{\mathrm{c}}=6047 \AA, F W H M=35 \AA\right)$ is comparable to the WR $r$ filter $\left(\lambda_{\mathrm{c}}=6000 \AA, F W H M=100 \AA\right)$. In some cases, line-free continua were below the detection limit of the WHT. Unfortunately, our Gemini GMOS and CFHT MOS imaging were not sufficiently deep for significant improvements upon this. In such cases, we were able to obtain estimates of the $v$-band magnitude via our GMOS spectrophotometry. Slit losses have not been taken into account, although agreement between narrow-band photometry and spectrophotometry is excellent, with $\mathrm{c} 1-v(\mathrm{GMOS}) \simeq 0.1 \mathrm{mag}$ on average. This is expected given the zero-point scale of the Royer et al. (1998) system.

We have also obtained $V$ broad-band photometry of 10 confirmed and 2 candidate WR stars from HST/WFPC2 images obtained with the F555W filter (10 exposures of $1400 \mathrm{~s}$ each; see Hunter 2001). The DAOPHOT package within IRAF was used to obtain instrumental magnitudes, which were then calibrated following Dolphin (2000a,b). We cross-checked our calibration by comparing Hunter's (2001) photometry of the dense clusters in the same images with our estimates, which agreed within $0.1 \mathrm{mag}$. In several cases, the ground-based image is resolved into multiple sources, which we shall refer to as A (brighter source) and B (fainter source). A careful comparison of the F814W ( $I$ band), F555W $(V)$ and F336W $(U)$ WFPC2 images (see Hunter 2001) shows that in all cases, star A is much bluer than star B. It is therefore very likely that star A is indeed the WR star in all cases. The only exception to this is WR24 from Massey \& Holmes (2002) which will be discussed separately. Again, for cases without HST $V$-band photometry, estimates were obtained from our GMOS spectrophotometry. For stars in common, $-0.3 \mathrm{mag} \leq V(\mathrm{HST})-V(\mathrm{GMOS}) \leq 0.3 \mathrm{mag}$.

We were also able to estimate interstellar reddenings from c1-c2 (i.e. $v-r)$, assuming an intrinsic colour of $(v-r)_{0}=$ -0.2 mag. Interstellar reddenings, $E_{b-v}$ are obtained via $E_{b-v} \sim$ $1.36 E_{v-r}$ using a standard extinction law. Note that groundbased photometry for [MAC92] 12 is heavily contaminated by 
nearby redder stars, as determined from UBV WFPC2 imaging, such that WHT photometry suggests a reddening which is far too high. The same is true for [MAC92] 14 such that we have set $E_{b-v}=1.0 \mathrm{mag}$ for these in Table 1, which is typical of other nearby stars.

\subsection{Metallicity of IC 10}

As discussed above, we were able to observe several H II regions of IC 10 with GMOS, with a view to re-determining the metallicity of this galaxy. Previously, Lequeux et al. (1979) obtained $\log (\mathrm{O} / \mathrm{H})+12=8.17$ for their $\mathrm{H}$ II region $\# 1$ and 8.45 for region \#2, whilst Garnett (1990) re-determined $\log (\mathrm{O} / \mathrm{H})+$ $12=8.26$, i.e. $0.25 Z_{\odot}$, for the latter ${ }^{1}$. This is intermediate between the oxygen content measured in the Small and Large Magellanic Clouds. More recently, Richer et al. (2001) determined a lower range of $\log (\mathrm{O} / \mathrm{H})+12=7.84-8.23$ for Hodge \& Lee (1990) H II regions $106 \mathrm{~b}$ and $111 \mathrm{~b} / \mathrm{c}$.

From the $144 \mathrm{H}$ II regions discussed by Hodge \& Lee, we have obtained GMOS spectroscopy for four - HL 16, 22, 45 and 128. In addition, we observed four candidate HII regions identified from narrow-band imaging by Royer et al. (2001). We employ [O III] $\lambda 4363 / \lambda 5007$ for $T_{\mathrm{e}}$ and [S II] $\lambda 6717 / \lambda 6731$ for $N_{\mathrm{e}}$. Unfortunately, far blue spectroscopy necessary for the measurement of [O II] $\lambda 3727$ is absent, such that merely lower limits to the oxygen abundance may be obtained. Sadly, [O III] $\lambda 4363$ lies below the detection threshold of most H II regions, so it ultimately proved difficult to determine nebular conditions from our present set of GMOS observations.

Observations of HL 22 imply $E(B-V)=0.99 \mathrm{mag}$ from the observed $F(\mathrm{H} \alpha) / F(\mathrm{H} \beta)$ ratio of 8.1, assuming a standard intrinsic ratio of 2.85. Nebular diagnostics imply $T_{\mathrm{e}}=$ $10800 \mathrm{~K}$ and $\log \left(N_{\mathrm{e}} / \mathrm{cm}^{-3}\right)=2$, such that the de-reddened $I([\mathrm{O}$ III $] 5007) / I(\mathrm{H} \beta)$ ratio of 3.31 reveals $\log \left(\mathrm{O}^{2+} / \mathrm{H}^{+}\right)+12=$ 7.97. From previous work, $\mathrm{O} / \mathrm{H} \sim 1.3 \mathrm{O}^{2+} / \mathrm{H}^{+}$(Lequeux et al. 1979), such that our observations of HL 22 suggest $\log (\mathrm{O} / \mathrm{H})+12 \sim 8.1$, comparable to other recent determinations.

\section{Properties of Wolf-Rayet stars}

We have used GMOS/MOS spectroscopy of Wolf-Rayet candidates in IC 10 to verify previous results, in many cases from narrow-band photometry alone. In most cases we have been able to confirm photometric spectral classifications, although there are some exceptions.

\subsection{New Catalogue}

The two previous narrow-band imaging surveys in IC 10 by Massey et al. (1992, [MAC92]) and Royer et al. (2001, RSMV) led to the discovery of 22 and 13 candidates, respectively. Of the 22 Massey et al. candidates, four were confirmed in the original paper, plus a further 11 were spectroscopically confirmed by Massey \& Armandroff (1995). New Gemini GMOS

\footnotetext{
1 We adopt $\log (\mathrm{O} / \mathrm{H})+12=8.83$ for the Solar abundance (Grevesse \& Sauval 1998).
}

spectroscopy for 14/15 WR stars from Massey et al. are presented here ${ }^{2}$. WR classifications are supported in all cases, although [MAC92] 5 is identified as a member of the rare WN/C intermediate subclass, due to exceptionally strong C IV 5801-12 $\AA$ emission. This is evident in the spectrum presented by Massey \& Armandroff (1995), and explains why narrowband photometry of Royer et al. (2001) suggested a WCE identification. We also obtained GMOS spectroscopy of two remaining candidate WR stars, \#3 and 16 from Massey et al., neither of which was confirmed as a Wolf-Rayet star.

The present study represents the first spectroscopic follow up to the 13 photometric candidates of Royer et al. (2001). Ten candidates were observed with Gemini GMOS, with the remainder (RSMV 1, 10 and 11) observed with CFHT MOS. In total, 9 stars were confirmed as WR stars, with the exception of all three WC9 candidates (RSMV 1, 3 and 7), plus the WC candidate RSMV 4.

Subsequent to the completion of our observing program, Massey \& Holmes (2002) have spectroscopically confirmed two other emission line stars in IC 10, although see Sect 3.2 with regard to the nature of \#24. In Table 1, we list 26 WR stars - as is usual ordered by increasing right ascension - that are either confirmed here with our new spectroscopy, or literature datasets firmly establish their nature. The present catalogue supersedes the earlier Massey et al. (1992) listing (see also Table 12 of Massey \& Johnson 1998). Note that "WR 4" from Massey et al. (1992), which is identified as IC 10 WR 4 by Massey \& Johnson (1998) should be referred to as [MAC92] 4. Similarly, candidate WR star \#6 from Royer et al. (2001) may be referred to as RSMV 6. Where high spatial resolution observations reveal multiplicity, we have denoted the components by A, B, etc. Use of this nomenclature is recommended to alleviate any possible confusion. Indeed, should large numbers of bonafide WR stars be identified in IC 10, as appears likely (Massey \& Holmes 2002), a master catalogue may eventually need to be be created.

We present finding charts for all confirmed WR stars in IC 10 in Fig. 1. These were obtained from our WHT He II $\lambda 4686$ narrow-band imaging and supersede earlier charts from Massey et al. (1992). In Fig. 2, we present HST WFPC2 images of 12 WR stars, revealing several that are resolved into multiple objects, A and B. In these instances, the Gemini GMOS slit contained both objects.

Absolute magnitudes, $M_{v}$, shown in Table 1 then follow from an adopted distance modulus (DM) of $23.86(d=$ $590 \mathrm{kpc}$ ), as discussed below. At this distance one arcsec subtends $\sim 3 \mathrm{pc}$. Interstellar reddenings and absolute magnitudes are rather uncertain, but typical values compare well with other Local Group WR stars (e.g. van der Hucht 2001). In general, stars which appear to be multiple spectroscopically, are indeed intrinsically bright, whilst those which may be single are faint, with the exception of RSMV 8 for which

\footnotetext{
${ }^{2}$ [MAC92] 15 was not observed with either Gemini or CFHT. Fortunately, the positive WR identification of this star is not in doubt, since Massey \& Armandroff (1995) spectroscopically demonstrate that this is a WC6-7 star.
} 


\begin{tabular}{|c|c|c|c|c|c|c|c|c|c|c|c|c|c|c|}
\hline \multirow{3}{*}{$\begin{array}{l}\text { Name } \\
\# \\
\text { [MAC92] } 1\end{array}$} & \multirow[t]{2}{*}{ RA } & \multirow{2}{*}{$\begin{array}{l}\text { Dec } \\
2000\end{array}$} & \multirow{3}{*}{$\begin{array}{l}V \\
\text { mag }\end{array}$} & \multirow{3}{*}{$\begin{array}{c}\begin{array}{c}v \\
\mathrm{mag}\end{array} \\
22.0\end{array}$} & \multirow{3}{*}{$\begin{array}{l}\begin{array}{l}E_{b-v} \\
\text { mag }\end{array} \\
1.02\end{array}$} & \multirow{3}{*}{$\begin{array}{c}\begin{array}{c}M_{v} \\
\mathrm{mag}\end{array} \\
-6.0\end{array}$} & \multirow{2}{*}{\multicolumn{2}{|c|}{$\begin{array}{c}\log W_{\lambda} F W H M \\
4650 / 4686\end{array}$}} & \multirow{2}{*}{\multicolumn{2}{|c|}{$\begin{array}{c}\log W_{\lambda} F W H M \\
\quad \text { CIV 5801-12 }\end{array}$}} & \multicolumn{3}{|c|}{ Spectral Type } & \multirow{3}{*}{$\begin{array}{l}\text { GMOS } \\
\text { Field }\end{array}$} \\
\hline & & & & & & & & & & & Photometric & Old & New & \\
\hline & 001956.97 & +591708.0 & & & & & 1.85 & 65 & 2.04 & 50 & WC & & WC4-5+abs & \\
\hline [MAC92] 2 & 001959.65 & +591655.3 & & 21.7 & 1.17 & -7.0 & 1.94 & 74 & 2.30 & 66 & WC? & WC & WC4 & Can, MOS \\
\hline RSMV 6 & 002003.02 & +591827.4 & $(22.5)$ & 22.8 & 1.22 & -6.1 & 2.25 & 78 & 2.33 & 82 & WC & & WC4 & Can \\
\hline RSMV 5 & 002004.24 & +591806.6 & $(22.0)$ & 22.4 & 1.44 & -7.4 & 2.09 & 79 & 2.11 & 80 & WC & & WC4-5+abs & Can, MOS \\
\hline [MAC92] 4 & 002011.55 & +591858.3 & (20.1) & 20.3 & 1.26 & -8.7 & 1.70 & 64 & 1.60 & 49 & WN? & WC & WC4-5 & Can \\
\hline [MAC92] 5 & 002012.85 & +592008.5 & $(22.4)$ & 23.2 & 1.14 & -5.3 & 2.62 & 53 & 3.10 & 79 & WN & WN & WNE/C4 & UK, Can \\
\hline RSMV 13 & 002015.62 & +591722.2 & $(23.8)$ & $(24.0)$ & $(0.7:)$ & $-2.8:$ & 2.36 & 27 & & & $\mathrm{WN}$ & & WN5 & UK \\
\hline RSMV 9 & 002020.33 & +591840.2 & $(22.0)$ & 21.6 & 1.16 & -7.0 & 1.49 & 28 & & & WN7 & & $\mathrm{WNE}+\mathrm{abs}^{c}$ & UK \\
\hline RSMV 8 & 002020.56 & +591837.8 & $(20.8)$ & 20.8 & 1.11 & -7.6 & 0.85 & 9 & & & WN8-9 & & WN10 & Can \\
\hline [MAC92] 7 & 002021.87 & +591741.5 & (19.4) & 19.6 & 1.01 & -8.4 & 1.95 & 84 & 1.91 & 82 & WN & WC & WC4-5+abs & Can \\
\hline [MAC92] 9 & 002022.60 & +591847.3 & 23.1 & (23.9) & $(0.8:)$ & -3.2 : & 2.38 & 30 & & & WN & $\mathrm{WN}$ & WN3 & UK \\
\hline RSMV 11 & 002022.68 & +591753.9 & 22.8 & & & & & & & & WC & & WC4 & MOS \\
\hline [MAC92] 10 & 002023.36 & +591742.6 & $(20.9)$ & 22.2 & 0.98 & -5.7 & 3.06 & 68 & 3.29 & 85 & WC & WC6-7 & WC7 & Can \\
\hline RSMV 12 & 002025.61 & +591648.6 & 22.7 & $(23.8)$ & $(1.3:)$ & $-5.4:$ & 2.31 & 68 & & & $\mathrm{WN}$ & & WNE & Can \\
\hline [MAC92] 12 & 002026.17 & +591726.9 & 21.9 & 22.0 & $1.0^{a}$ & -6.0 & 1.86 & 58 & 1.84 & 49 & WN? & WC & $\mathrm{WC} 4+\mathrm{a}$ & UK \\
\hline RSMV 10 & 002026.48 & +591705.3 & 22.9 & & & & & & & & WC & & WC4 & MOS \\
\hline [MAC92] 13-A & 002026.63 & +591733.2 & 20.8 & 21.4 & 1.03 & -6.7 & 2.07 & 58 & 2.04 & 47 & WC & WC & WC5-6 & Can \\
\hline [MAC92] 13-B & 002026.62 & +591733.4 & 23.1 & & & & & & & & & & & \\
\hline [MAC92] 14-A & 002026.87 & +591720.2 & 20.7 & 21.4 & $1.0^{a}$ & -6.6 & 2.67 & 79 & 2.47 & 78 & WC & WC & WC5 & UK \\
\hline [MAC92] 14-B & 002026.91 & +591720.3 & 22.4 & & & & & & & & & & & \\
\hline [MAC92] 15 & 002027.03 & +591818.6 & & 23.3 & 1.05 & -4.9 & & & & & WN? & WC6-7 & & \\
\hline [MAC92] 24-A & 002027.67 & +591737.7 & 18.8 & & & & 0.6: & 21 & & & & WN & $\mathrm{WN}+\mathrm{OB}^{c}$ & \\
\hline [MAC92] 24-B & 002027.82 & +591737.5 & 21.3 & & & & & & & & & & & \\
\hline [MAC92] 24-C & 002027.75 & +591736.3 & 22.0 & & & & & & & & & & & \\
\hline RSMV 2-A & 002028.00 & +591714.6 & 21.4 & 22.4 & 1.24 & -6.5 & 1.53 & 17 & & & WN7-8 & & WN7-8 & UK \\
\hline RSMV 2-B & 002028.04 & +591714.9 & 25.1 & & & & & & & & & & & \\
\hline [MAC92] 17-A & 002029.05 & +591652.3 & 22.5 & & & & 1.5: & 15 & & & WN & WN & $\mathrm{WNE}+\mathrm{OB}$ & Can \\
\hline [MAC92] 17-B & 002028.98 & +591651.9 & 22.2 & & & & & & & & & & & \\
\hline [MAC92] 19-A & 002031.01 & +591904.5 & 22.6 & 23.3 & 1.32 & -6.0 & 2.07 & 25 & & & $\mathrm{WN}$ & WNE & WN4 & UK, Can \\
\hline [MAC92] 19-B & 002031.01 & +591905.2 & 24.0 & & & & & & & & & & & \\
\hline [MAC92] 23 & 002032.79 & +591716.4 & & & & & 1.60 & 13 & & & & WN7-8 & & \\
\hline [MAC92] 20 & 002034.46 & +591714.7 & 22.0 & $(22.8)$ & $(0.7:)$ & $-3.9:$ & 2.95 & 62 & 2.86 & 52 & WC & WC & WC5 & UK \\
\hline [MAC92] 21 & 002041.64 & +591625.3 & & 23.4 & 0.76 & -3.6 & 2.44 & 26 & & & $\mathrm{WN}$ & $\mathrm{WN}$ & WN4 & UK \\
\hline
\end{tabular}

(a): Set at $E_{b-v}=1.0$ due to severe contamination of ground based photometry by nearby red stars (see text)

(b) RSMV 9 does not appear to be spatially coincident with [MCA92] 6 - see Fig. 1.

(c) Spectral type re-evaluated based on published spectroscopy of Massey \& Holmes (2002, see Sect 3.2). 

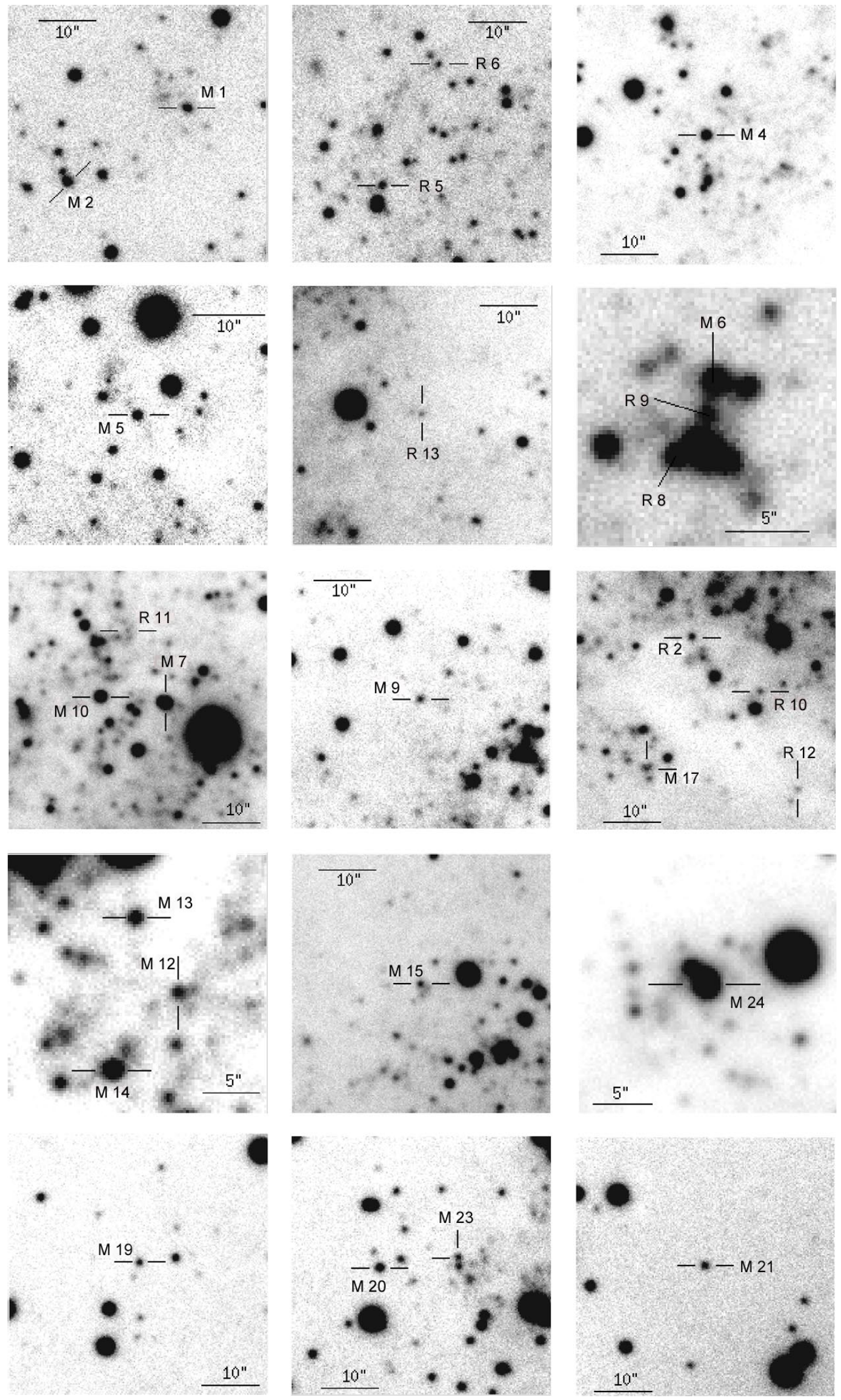

Fig. 1. Finding charts (typically $45^{\prime \prime} \times 45^{\prime \prime}$ ) for WR stars in IC 10 based on WHT He II narrow-band imaging. Stars listed in the present work are indicated with either M \# (MAC92) or R \# (RSMV). [MAC92] 6 is spatially close (a few arcsec away) from the confirmed WR stars RSMV 9 and RSMV 8, but is not apparently coincident (despite suggestions to the contrary by Massey \& Holmes 2002). North is up and east is to the left. 
$M_{v}(\mathrm{WN} 10)=-7.6 \mathrm{mag}$ is realistic when compared with other very late WN stars (Crowther \& Smith 1997).

Our adopted distance was derived from near-IR photometry by Borissova et al. (2000), with respect to IC 1613, which possesses a more robust distance modulus (Freedman et al. 2001). This lies between the two distance moduli obtained by Sakai et al. (1999) from Cepheid variables (DM $=24.1$, $d=660 \mathrm{kpc})$ and from the tip of the RGB, (DM $=23.5$, $d=500 \mathrm{kpc}$ ). Clearly, the high and variable extinction towards IC 10 favours IR techniques which are less susceptible to reddening. Our present, imprecise, method of determining reddenings favours the Borissova et al. distance, whilst lower reddenings (e.g. from nebular methods) may favour higher distance scales (e.g. $\mathrm{DM}=24.57, d=820 \mathrm{kpc}$ : Kennicutt et al. 1998).

\subsection{WN stars}

We first discuss two stars that have previously been classified as WN stars, yet their exact nature is in doubt, given their weak reported He II emission line strengths. Ultimately, the dividing line between O3 If/WN stars, WN9-11 stars and normal WN stars is rather arbitrary, with $W_{\lambda}(\mathrm{He}$ II 4686) $>10-12 \AA$ in most cases (see Crowther \& Dessart 1998). Nevertheless, one needs to ensure consistency when comparing the population of emission line stars in external galaxies, or else e.g. WC/WN ratios would be artificially skewed in favour of WN stars.

[MAC92] 17 is classified as a WN star according to Massey \& Armandroff (1995), despite "very weak emission" at He II 4686. Our initial GMOS extraction confirmed He II $\lambda 4686$ emission, albeit with an equivalent width of only $\sim 8 \AA$, such that [MAC92] 17 most closely resembled an O3 If/WN star. Alternatively, the stellar continuum of a genuine WN star may be heavily contaminated by a line-of-sight or binary companion. One might suspect the latter case given the large distance to IC 10, so we have inspected HST/WFPC2 images which reveal two sources, -A and -B, within $1^{\prime \prime}$ of the coordinates of Massey \& Holmes (2002, see Fig. 2). Therefore, we subsequently re-examined our raw frames and discovered that He II emission spanned only half of the stellar continuum. We carefully re-extracted this narrow ( $\sim 5$ pixel) region, which now revealed a significantly stronger He II $\lambda 4686$ emission of $\sim 30 \AA$, with weak N v $\lambda \lambda 4603-30$ emission also present. This spectrum in the vicinity of $\lambda 4686$ is presented in Fig. 3 and so [MAC92] 17-A is clearly consistent with a WNE star.

There is another weak He II emission line star in IC 10, which has recently been classified as a WN star, namely WR24 from Massey \& Holmes (2002), hereafter [MAC92] 24 (following the CDS nomenclature - see Sect 3.1). We have not obtained new spectroscopy of this star in the present program, given its location within the bright $\mathrm{H}$ II region $\mathrm{HL} 111 \mathrm{c}$ (Hodge \& Lee 1990) ${ }^{3}$. [MAC92] 24 was not identified as a

\footnotetext{
${ }^{3}$ Note that the putative WN star identified by Richer et al. (2001) in HL111c is most likely [MAC92] 24. Richer et al. described a WN star with $W_{\lambda}(\mathrm{He}$ II 4686) $=3 \AA$, consistent with Massey \& Holmes (2002), and their coordinates agree to within 2 arcsec, comparable to the slit width used in the former.
}

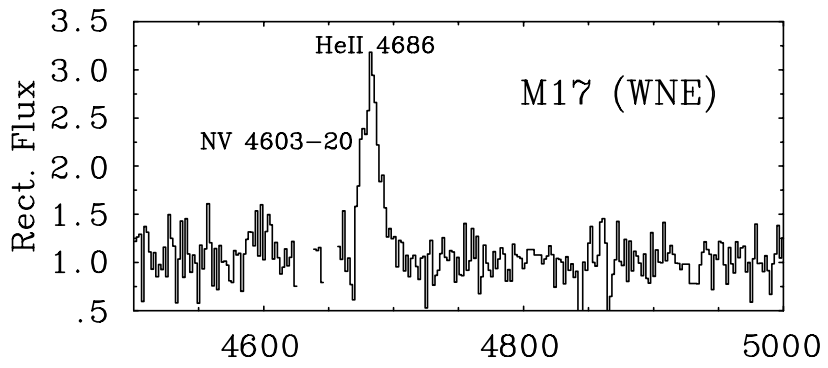

Fig. 3. GMOS spectroscopy of [MAC92] 17 from Massey et al. (1992), in the vicinity of He II $\lambda 4686$ indicating a WNE spectral type.

He II emission line source in Royer et al. (2001) and its nature is also in doubt.

Spectroscopically, the published data for [MAC92] 24 most closely resembles an O3 If WN star, such as Melnick 42 in the LMC, which has a higher He II $\lambda 4686$ equivalent width, plus a similar FWHM. Massey \& Holmes (2002) favoured a WN binary nature given that it was exceptionally bright. Therefore, we have again inspected HST/WFPC2 F555W images, which reveals three sources within 1 " from the published coordinates of [MAC92] 24, as indicated in Fig. 2. The ground-based slit spectrum will have contributions from each, with the continuum dominated by source A $(V \sim 18.8 \mathrm{mag})$. All are consistent with early-type stars, with similar $U-V$ colours, preventing any further discrimination of the true emission line source. The severe crowding of this region is apparent from the figure, with two further bright sources lying within $2^{\prime \prime}$, to the $\mathrm{N}$ $(V \sim 19.4 \mathrm{mag})$ and NE $(V \sim 19.8 \mathrm{mag})$. From a combination of the existing spectroscopy and imaging, one would favour a $\mathrm{WN}+\mathrm{OB}$ binary, or a perhaps a cluster containing a WN star.

If this is the case, one puzzle remains. The high $S / N$ spectroscopy of [MAC92] 24 presented by Massey \& Holmes (2002) shows no sign of N III-V $\lambda \lambda 4603-40$ emission, which would be expected if this was indeed a $\mathrm{WN}+\mathrm{OB}$ binary. Undoubtedly, higher spatial resolution imaging/spectroscopy is needed for WR confirmation of this object, but for the moment we adopt $\mathrm{WN}+\mathrm{OB}$ for its spectral type despite the lack of N III-V emission.

Let us now turn to the more conventional WN stars in IC 10. Figure 4 presents Gemini GMOS spectroscopy of eight WN stars. Precise WN spectral types are rather difficult to establish since spectroscopy for most stars is limited to regions redward of N IV 24058 . Nevertheless, including [MAC92] 23 from Massey \& Holmes (2002), IC 10 hosts seven early (2-5) WN stars and three late (6-11) WN stars, plus [MAC92] 24 for which we have no subtype information. Amongst the WNE stars, He II $\lambda 4686$ emission is extremely weak in RSMV 9 and RSMV 12. The latter might be a binary. WFPC2 imaging reveals no nearby line-of-sight companions contaminating our Gemini spectroscopy. The remainder are potentially single, and have line strengths and widths comparable to LMC WNE stars. RSMV 2 and [MAC92] $23^{4}$ are late WN7-8 stars with

\footnotetext{
4 Royer et al. (2001) identified [MAC92] 23 as a strong He II $\lambda 4686$ emission source, but it was excluded from their catalogue on the basis of a very red colour $(c 1-c 2>1 \mathrm{mag})$, common to many foreground late-type stars.
} 

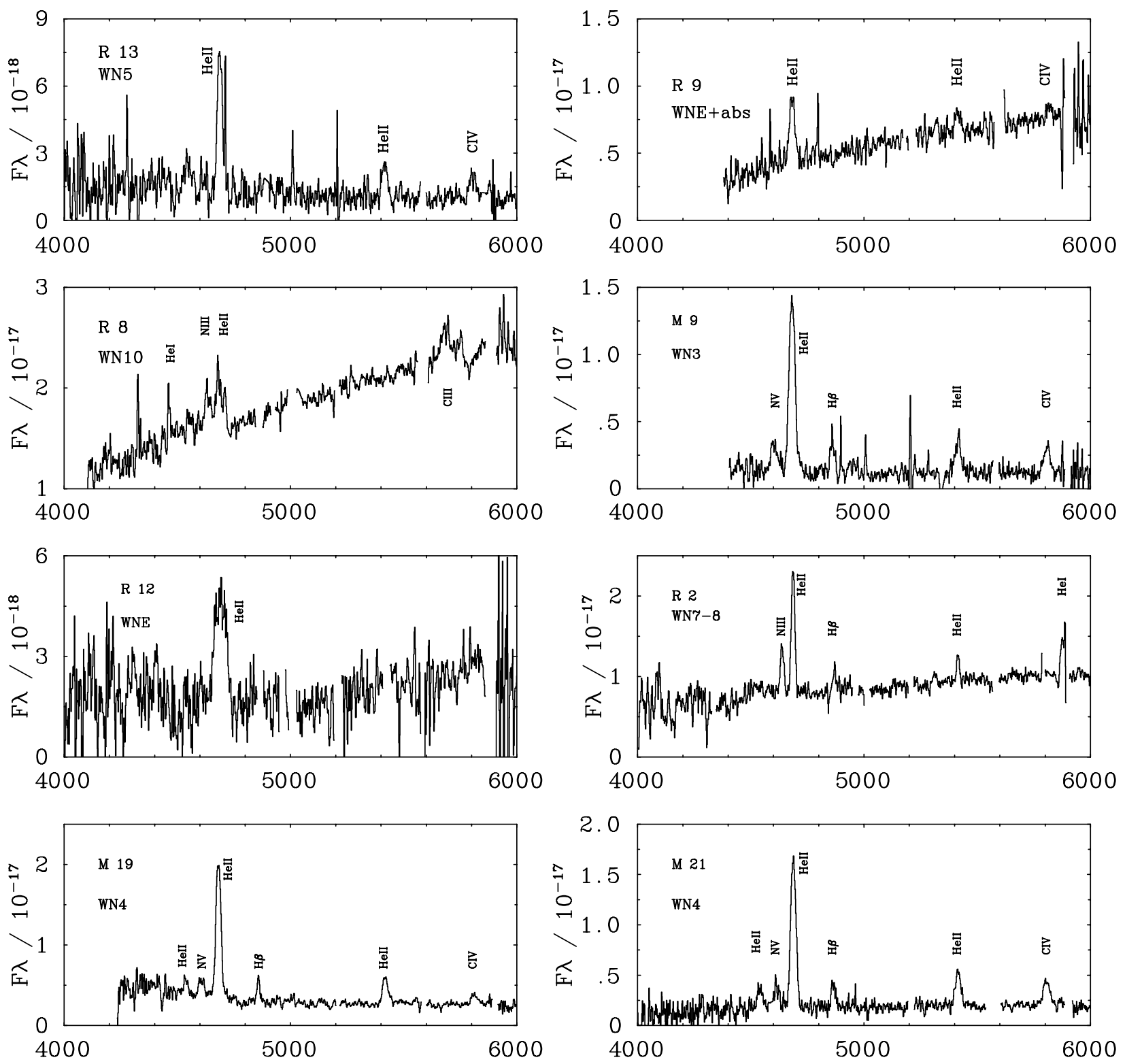

Fig. 4. Flux calibrated (erg cm $\mathrm{cm}^{-2} \mathrm{~s}^{-1} \AA^{-1}$ ) optical spectroscopy of WN stars in IC 10 observed with Gemini GMOS.

relatively strong N III $\lambda \lambda 4634-40$ emission, although the former has a faint companion as revealed by WFPC 2 imaging. RSMV 8 is another unusual example of a very late WN10 star (e.g. Crowther \& Smith 1997).

For comparison, the Solar Neighbourhood hosts a similar fraction of WNE and WNL stars (van der Hucht 2001), whilst WNE stars outnumber WNL stars by approximately 5:2 in the LMC (Breysacher et al. 1999), and there are no WNL stars in the SMC (Massey \& Duffy 2001), such that the WN population of IC 10 is closer to the LMC than the SMC. Crowther (2000) explained the general trend to earlier spectral type amongst WN stars at lower metallicity due to the differing dependence of nitrogen emission lines on reduced $\mathrm{CNO}$ equilibrium abundances. Indeed, in contrast with Galactic late WN stars, there are no IC10 cases in which N III $\lambda \lambda 4640$ is comparable in strength to He II 14686 , an effect remarked upon already for the LMC by Crowther \& Smith (1997).

\subsection{WN/C and WC stars}

Figure 5 presents Gemini GMOS spectrophotometry of twelve WC stars, including [MAC92] 5 which is newly identified here as a WNE/WC4 star, whilst Fig. 6 shows MOS spectroscopy for RSMV 10 and 11. It is well known that late type (WC8-9) stars are only observed in metal-rich environments, such as the inner Milky Way and M31 (van der Hucht 2001). Indeed, of the 14 WC stars in IC 10, at least twelve are WC4-6 subtypes. [MAC92] 10 is a notable exception, i.e. a WC7 star with extremely broad lines $\left(v_{\infty} \sim 3000 \mathrm{~km} \mathrm{~s}^{-1}\right)$. [MAC92] 15 was listed as WC6-7 by Massey \& Armandroff (1995), though their Fig. 1 appears to rule out a subtype as late as WC7 for this 

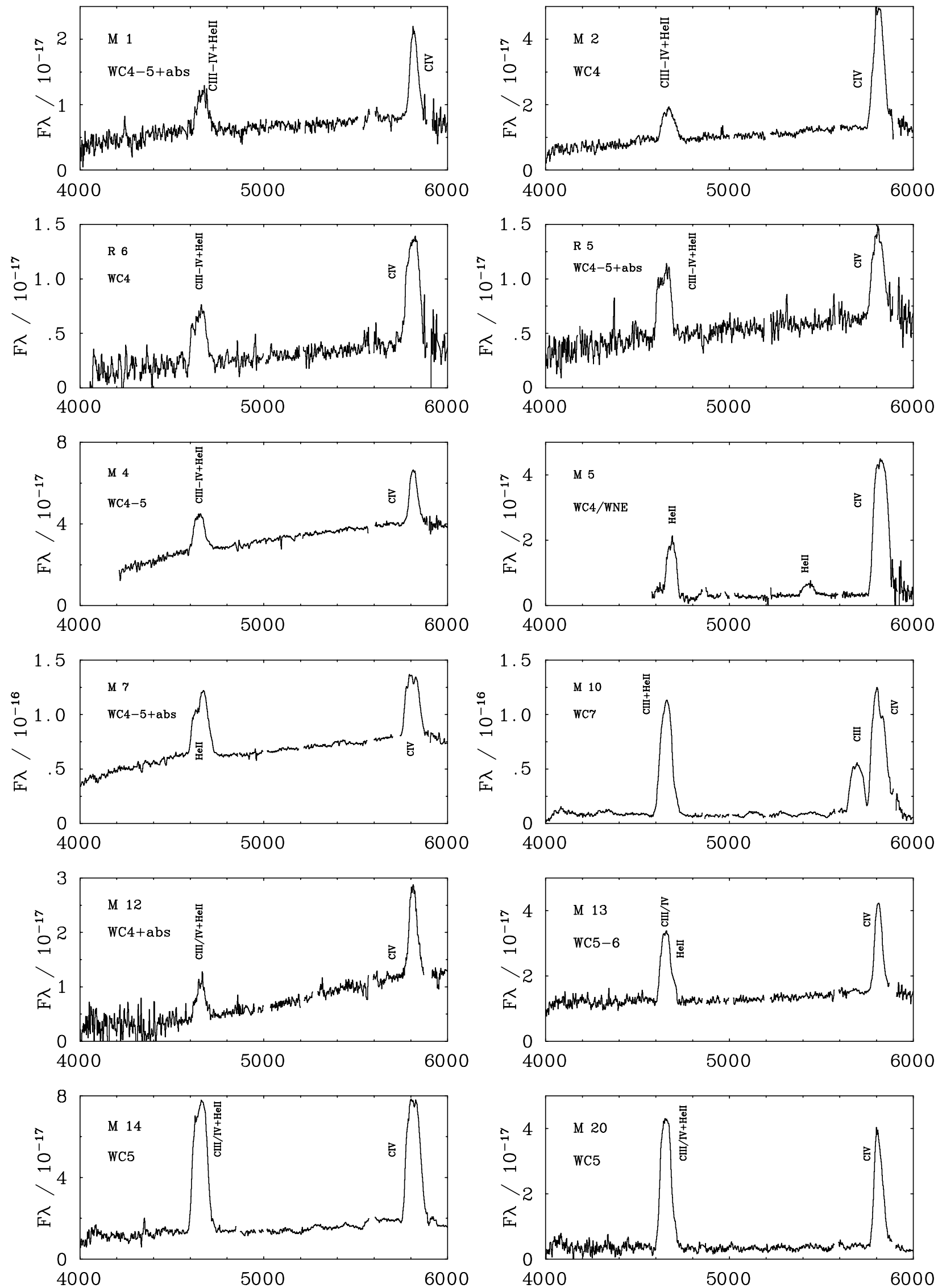

Fig. 5. Flux calibrated $\left(\mathrm{erg} \mathrm{cm} \mathrm{cm}^{-2} \mathrm{~s}^{-1} \AA^{-1}\right)$ optical spectroscopy of WN/C and WC stars in IC 10 observed with Gemini GMOS. 

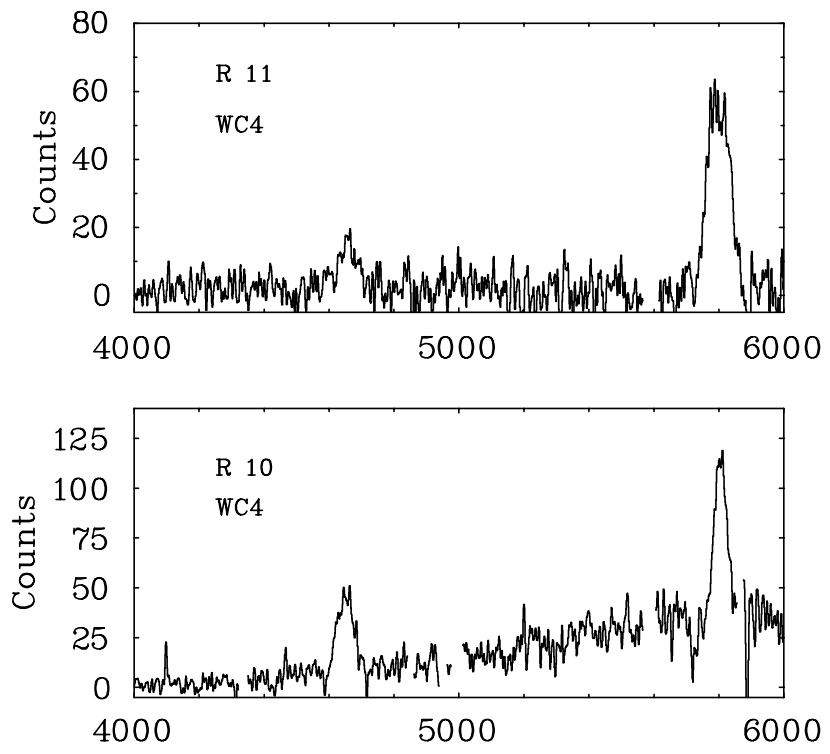

Fig. 6. Optical spectroscopy of WC stars in IC 10 observed with CFHT MOS.

star. Consequently, [MAC92] 10 appears to be unique amongst low metallicity WC stars in having relatively strong C III $\lambda 5696$ emission. Crowther et al. (2002) recently claimed to have established the origin of the WC subtype distribution amongst Local Group galaxies. They established a metallicity dependence amongst WC winds, reminiscent of OB stars, with the strength of C III $\lambda 5696$ exceptionally sensitive to wind density. The dominance of WCE stars within the low metallicity environment of IC 10 naturally follows this pattern, with the conspicuous exception of [MAC92] 10, which is remarkably similar to V378 Vul (alias WR125) in our Galaxy.

In contrast with the WN population, the majority of WC stars are heavily contaminated by OB line-of-sight or binary light. This is demonstrated in Fig. 7 where we compare the C IV 15801-12 emission line strengths and estimated absolute visual magnitudes from our ground-based photometry of IC 10 WC stars versus LMC WC single star and binaries (Smith et al. 1990). From this comparison, [MAC92] 10 and 20 appear to be convincingly single, or at least the lines and continua are not strongly diluted. All others suffer considerable contamination in their spectra. More IC $10 \mathrm{WC}$ stars may be single, but higher angular resolution observations would be required. Indeed, corrections for line-of-sight close companions are shown in Fig. 7 for [MAC92] 12, 13 and 14 based on WFPC2 imaging.

Nevertheless, cases such as [MAC92] 10 and 20 are important, since it is commonly assumed that binary evolution (via Roche Lobe overflow) is required for evolution to late Wolf-Rayet phases at low metallicity. For instance, the only carbon/oxygen sequence WR star in the SMC, Sand 1 (WO+O4V), has a close massive companion (Moffat et al. 1985). Therefore, the presence of (at least) two such cases in IC 10 suggests that progression to late phases via single stellar evolution may be achieved at low metallicities. The difference with respect to the SMC may solely be as a result of a much larger massive stellar population, such that there will

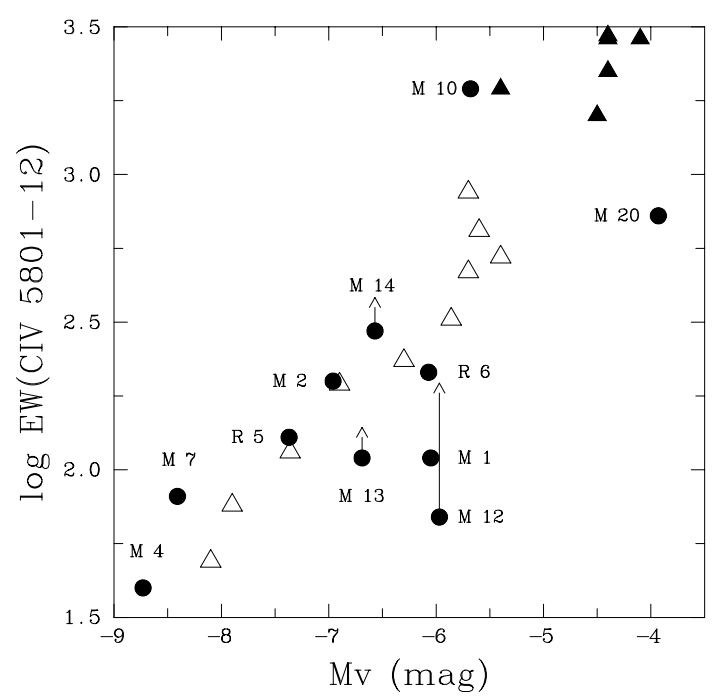

Fig. 7. Comparison between the emission C IV $\lambda 5801-12$ emission equivalent widths (in $\AA$ ) measured in our Gemini and CFHT datasets and absolute visual magnitude estimates for IC $10 \mathrm{WC}$ stars (filled circles). Corrections to measured GMOS equivalent widths are shown for three stars, owing to contamination by nearby companions from HST WFPC2 images. Single (filled triangles) and binary (open triangles) WC stars in the LMC are shown for comparison (taken from Smith et al. 1990) assuming an LMC distance modulus of 18.50 .

be a higher statistical likelyhood of extremely massive stars in IC 10, under the assumption of a universal Salpeter IMF.

Finally, we have compared the C IV $\lambda \lambda 5801-12$ line flux in IC 10 WC stars with those of LMC WC stars. Smith et al. (1990) claimed that LMC early type WC stars possessed uniform $\lambda \lambda 5801-12$ line fluxes of $\log F_{\lambda}=-7.6 \pm 0.1 \mathrm{erg} \mathrm{s}^{-1} \mathrm{~cm}^{-2}$. Despite the imprecise reddenings to many IC $10 \mathrm{WC}$ stars, we obtain an essentially identical mean line flux, $\log F_{\lambda}=$ $-7.5_{-0.6}^{+0.2} \mathrm{erg} \mathrm{s}^{-1} \mathrm{~cm}^{-2}$. Firmer conclusions relating to a universal early WC await more reliable reddening determinations from HST.

\section{4. $W C / W N$ ratio for IC 10}

What effect does our new spectroscopic results have on the unusual WC/WN ratio for IC 10? Our datasets, together with previous spectroscopy imply $\mathrm{WC} / \mathrm{WN}=14 / 11 \sim 1.3$, where we have omitted the intermediate star [MAC92] 5 from this ratio. Comparison with the WC/WN ratios for other Local Group galaxies in Fig. 8 would lead one to conclude that WC/WN 0.15 is anticipated for IC 10 . Clearly, for IC 10 to mimic other Local Group galaxies, another $\sim 80 \mathrm{WN}$ stars would be expected. According to WHT imaging by Royer et al. (2001) this is not likely, but Massey \& Holmes (2002) claim up to $\sim 75 \mathrm{WR}$ stars still await confirmation.

One has to bear in mind that the exact number expected depends heavily upon the strength of the excess measured in the WR filter relative to the continuum filter by Massey \& Holmes (2002). For example, examination of their Fig. 3 implies that $\sim 15$ have excesses greater than $0.5 \mathrm{mag}$, most of which are probably genuine WN stars, from comparison with their control field. Taking these into account would reduce the WC/WN 


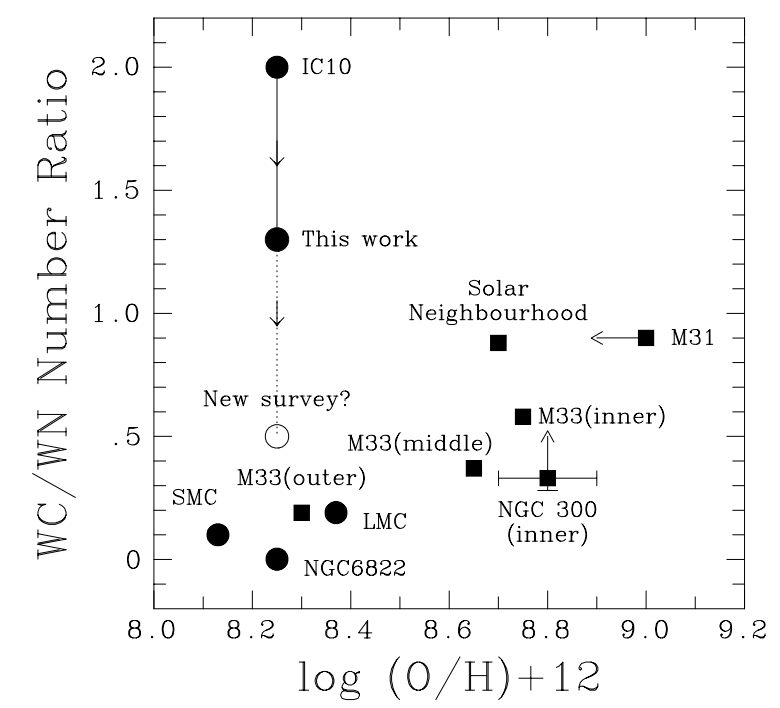

Fig. 8. WC/WN ratio for Local Group and Sculptor Group spiral (squares) and irregular galaxies (circles) versus oxygen content. Individual data points are taken from Massey \& Johnson (1998), Schild et al. (2003). Recent evidence, based on model atmosphere calculations rather than $\mathrm{H}$ II region analyses, suggests a lower metallicity for M31 (Trundle et al. 2002). The ratio for IC 10 is shown based on the Massey \& Johnson (1998) and present spectroscopic results (filled circles), plus a re-assessment of recent narrow-band imaging of Massey \& Holmes (2002, open circle).

ratio to $\sim 0.5$, as indicated with an open circle in Fig. 8 . This would make IC 10 unusually rich in WC stars for a low metallicity galaxy. In contrast, Massey \& Holmes were rather bolder, claiming instead $\mathrm{WC} / \mathrm{WN} \sim 0.3$. This was on the basis that all stars with excesses between 0.3-0.5 mag are bona fide WR stars, even though several stars in their control field had such excesses. Their justification for this conclusion was heavily reliant on [MAC92] 24 being a genuine WN star, since its excess lay at the $\sim 0.3$ mag limit. As we have discussed above, this object would not ordinarily be classified as a genuine WR star from a spectroscopic viewpoint, since (individual) stars with an emission equivalent width of $\sim 4 \AA$ at He II $\lambda 4686$ are most likely to be Of supergiants. In the case of [MCA92] 24, we consider it to be a probable WN star on the basis of additional information afforded by HST imaging, namely its brightness and apparent multiplicity. Consequently, a sizeable fraction of stars in IC 10 with such a small excess identified by Massey \& Holmes are likely to be Of, Of/WN stars or spurious candidates.

In fairness, the situation discussed above for IC 10 is common to all galaxies beyond the Magellanic Clouds, such that one has to be wary when comparing WC/WN ratios. There are cases of spectroscopically confirmed WRs stars in other Local Group galaxies with magnitude differences as low as $0.2 \mathrm{mag}$, although the vast majority greatly exceed 0.5 mag (e.g. Fig. 4 of Massey \& Johnson 1998). A handful of genuine WR stars are known for which He II emission strengths do not exceed 10-12 A, namely: (i) WR+OB binaries with extreme light ratios, such as Sk 108 in the SMC (e.g. Foellmi et al. 2003), although it should be noted that this is the only $\mathrm{WN}+\mathrm{OB}$ binary in the SMC for which He II $\lambda 4686$ does not exceed $10 \AA$; (ii) very late WN stars, such as RSMV 8 in IC 10 , have very small He II $\lambda 4686$ emission equivalent widths, which are similar to extreme Of stars (Crowther \& Bohannan 1997). Such stars are, generally, rather rare relative to Of stars.

For the moment, the jury remains out until all new candidates from Massey \& Holmes (2002) are spectroscopically observed. Ideally, the presence of photospheric absorption lines would be sought to resolve current uncertainties. More realistically, given the visual faintness and slit contamination, the strength of emission line fluxes might be used to verify WR versus Of/WN identifications. For the moment, we merely suggest that the WC/WN ratio of IC 10 may not be as low as $\sim 0.3$, as has been claimed by Massey \& Holmes (2002). Consequently, it is possible that the number and distribution of the WR population for IC 10 remains unusually high, relative to other Local Group irregular galaxies, as further evidence of a short, co-eval burst of star formation in the recent past.

In summary, we show that IC 10 hosts a substantial population of Wolf-Rayet stars. At present, spectroscopically confirmed WC stars outnumber WN stars, and early subtypes of both flavours outnumber late subtypes. Recent candidate WC9 stars are not confirmed, and remain exclusive to metal-rich environments, although the presence of [MAC92] 10, an apparently single, broad lined WC7 star was not anticipated in an environment of $0.25 Z_{\odot}$. This star illustrates that highly evolved Wolf-Rayet populations can occur in low metallicity environments without mass transfer in a close binary. WN stars are reminiscent of the LMC, with a large WN3-4 population, plus a few late ([MAC92] 23, WN7-8) and very late (RSMV 8, WN10) subtypes. Work is underway to analyse a subset of these objects, for comparison with counterparts in Local Group galaxies spanning a range of metallicities. Spectroscopy of all potential candidates from Massey \& Holmes (2002) is keenly sought in order to resolve remaining questions.

Acknowledgements. Based on observations obtained at the Gemini Observatory, which is operated by the Association of Universities for Research in Astronomy, Inc., under a cooperative agreement with the NSF on behalf of the Gemini partnership: the National Science Foundation (United States), the Particle Physics and Astronomy Research Council (United Kingdom), the National Research Council (Canada), CONICYT (Chile), the Australian Research Council (Australia), CNPq (Brazil), and CONICET (Argentina). Part of the data were obtained with the Canada-France-Hawaii Telescope, which is operated by the National Research Council of Canada, the Centre National de la Recherche Scientifique de France, and the University of Hawaii. Thanks to Inger Jorgensen for help with extraction of the Gemini datasets, to Pierre Martin for his contribution to the CFHT observations, and to Cedric Foellmi for providing spectroscopy of Sk 108 and Melnick 42. PAC and SJS acknowledge financial support from the Royal Society and PPARC respectively, and LD acknowledges NSERC and the Canada Research Chair Program

\section{References}

Armandroff, T. E., \& Massey, P. 1985, ApJ, 291, 685

Borissova, J., Georgiev, L., \& Rosado, M., et al. 2000, A\&A, 363, 130 Breysacher, J., Azzopardi, M., \& Testor, G. 1999, A\&AS, 137, 117

Crowther, P. A. 2000, A\&A 356, 191 
Crowther, P. A., \& Bohannan, B. 1997, A\&A, 317, 532

Crowther, P. A., \& Smith, L. J. 1997, A\&A, 320, 500

Crowther, P. A., \& Dessart, L. 1998, MNRAS, 296, 622

Crowther, P. A., Dessart, L., \& Hillier, D.J., et al. 2002, A\&A, 392, 653

Dolphin, A. E. 2000a, PASP, 112, 1383

Dolphin, A. E. 2000b, PASP, 112, 1397

Foellmi, C., Moffat, A. F. J., \& Guerrero, M. A. 2003, MNRAS, 338, 360

Freedman, W. L., Madore, B. F., \& Gibson, B. K., et al. 2001, ApJ, 553,47

Garnett, D. R. 1990, ApJ, 363, 142

Grevesse, N., \& Sauval, A. J. 1998, Space Sci. Rev., 85, 161

Hodge, P., \& Lee, M. G. 1990, PASP, 102, 26

Hook, I., Allington-Smith, J. R., Beard, S., et al. 2002, Proc SPIE, 4841

Hunter, D. A. 2001, ApJ, 559, 225

Kennicutt, R. C. Jr, Stetson, P. B., Saha, A., et al. 1998, ApJ, 498, 181

Lequeux, J., Peimbert M., Rayo, J. F., Serrano, A., \& Torres-Peimbert, S. 1979, A\&A, 80, 155

Massey, P. 2003, in Proc. IAU Symp 212, ed. K. A. van der Hucht et al., ASP, 316
Massey, P., \& Armandroff, T. E. 1995, AJ, 109, 2470

Massey, P., \& Duffy, A. S. 2001, ApJ, 550, 713

Massey, P., \& Holmes, S. 2002, ApJ, 580 L35

Massey, P., Armandroff, T. E., \& Conti, P. S. 1992, AJ, 103, 1159

Mellise, J. P., \& Israel, F. P. 1994, A\&AS, 103, 391

Moffat, A. F. J., Breysacher, J., \& Seggewiss, W. 1985, ApJ, 292, 511

Richer, M. G., Bullejos, A., Borissova, J., et al. 2001, A\&A, 370, 34

Royer, P., Vreux, J.-M., \& Manfroid, J. 1998, A\&AS, 130, 407

Royer, P., Smartt, S. J., Manfroid, J., \& Vreux, J.-M. 2001, A\&A, 366, L1

Sakai, S., Madore, B. F., \& Freedman, W. L. 1999, ApJ, 511, 671

Schaerer, D., \& Vacca, W. D. 1998, ApJ, 497, 618

Schild, H., Crowther, P. A., Abbott, J. B., \& Schmutz, W. 2003, A\&A, 397,859

Smith, L. F. 1968, MNRAS, 140, 409

Smith, L. F., Shara, M. M., \& Moffat, A. F. J. 1990, ApJ, 358, 229

Trundle, C., Dufton, P. L., Lennon, D. J., Smartt, S. J., \& Urbaneja, M. A. 2002, A\&A, 395, 519

van der Hucht, K. A. 2001, New Astron. Rev., 45, 135

Yang, H., \& Skillman, E. D. 1993, AJ, 106, 1448 